\title{
A Four-Month Gatifloxacin-Containing Regimen for Treating Tuberculosis
}

\author{
Corinne S. Merle, M.D., Katherine Fielding, Ph.D., Omou Bah Sow, M.D., \\ Martin Gninafon, M.D., Mame B. Lo, M.D., Thuli Mthiyane, M.Sc., \\ Joseph Odhiambo, M.D., Evans Amukoye, M.D., Boubacar Bah, M.D., \\ Ferdinand Kassa, M.D., Alimatou N'Diaye, M.D., Roxana Rustomjee, M.D., \\ Bouke C. de Jong, M.D., Ph.D., John Horton, M.D., Christian Perronne, M.D., \\ Charalambos Sismanidis, Ph.D., Olivier Lapujade, B.Sc., \\ Piero L. Olliaro, M.D., Ph.D., and Christian Lienhardt, M.D., Ph.D., \\ for the OFLOTUB/Gatifloxacin for Tuberculosis Project*
}

A BSTRACT

From the London School of Hygiene and Tropical Medicine, London (C.S.M., K.F., C.S.), and Tropical Projects, Hitchin (J.H.) - both in the United Kingdom; Service de Pneumo-phtisiologie, Hôpital Ignace Deen, Conakry, Guinea (O.B.S., B.B.); Centre National Hospitalier de Pneumophtisiologie, Cotonou, Benin (M.G., F.K.); Programme National de Lutte contre la Tuberculose, Dakar, Senegal (M.B.L., A.N.); Medical Research Council, Durban, South Africa (T.M., R.R.); Kenya Medical Research Institute, Nairobi (J.O., E.A.); Institute of Tropical Medicine, Antwerp, Belgium (B.C.J.); Hôpitaux Universitaires Paris Ile-de-France Ouest, Assistance Publique-Hôpitaux de Paris, Paris (C.P.), and Institut de Recherche pour le Développement, Marseille (O.L., C.L.) - both in France; and Special Program for Research and Training in Tropical Diseases, World Health Organization, Geneva (P.L.O.). Address reprint requests to Dr. Olliaro at the Special Program for Research and Training in Tropical Diseases, World Health Organization, 20 Ave. Appia, $\mathrm{CH}-1211$, Geneva 27, Switzerland, or at olliarop@who.int.

*Additional members of the OFLOTUB/ Gatifloxacin for Tuberculosis Project team are listed in the Supplementary Appendix, available at NEJM.org.

Drs. Olliaro and Lienhardt contributed equally to this article.

This article was updated on April 23, 2015, at NEJM.org.

N EnglJ Med 2014;371:1588-98. DOI: 10.1056/NEJMoa1315817

Copyright (C) 2014 Massachusetts Medical Society.

\section{BACKGROUND}

Shortening the course of treatment for tuberculosis would be a major improvement for case management and disease control. This phase 3 trial assessed the efficacy and safety of a 4-month gatifloxacin-containing regimen for treating rifampinsensitive pulmonary tuberculosis.

METHODS

We conducted a noninferiority, randomized, open-label, controlled trial involving patients 18 to 65 years of age with smear-positive, rifampin-sensitive, newly diagnosed pulmonary tuberculosis in five sub-Saharan African countries. A standard 6-month regimen that included ethambutol during the 2-month intensive phase was compared with a 4-month regimen in which gatifloxacin (400 mg per day) was substituted for ethambutol during the intensive phase and was continued, along with rifampin and isoniazid, during the continuation phase. The primary efficacy end point was an unfavorable outcome (treatment failure, recurrence, or death or study dropout during treatment) measured 24 months after the end of treatment, with a noninferiority margin of 6 percentage points, adjusted for country.

\section{RESULTS}

A total of 1836 patients were assigned to the 4-month regimen (experimental group) or the standard regimen (control group). Baseline characteristics were well balanced between the groups. At 24 months after the end of treatment, the adjusted difference in the risk of an unfavorable outcome (experimental group [21.0\%] minus control group [17.2\%]) in the modified intention-to-treat population (1356 patients) was 3.5 percentage points ( $95 \%$ confidence interval, -0.7 to 7.7$)$. There was heterogeneity across countries $(\mathrm{P}=0.02$ for interaction, with differences in the rate of an unfavorable outcome ranging from -5.4 percentage points in Guinea to 12.3 percentage points in Senegal) and in baseline cavitary status ( $\mathrm{P}=0.04$ for interaction) and body-mass index $(\mathrm{P}=0.10$ for interaction). The standard regimen, as compared with the 4-month regimen, was associated with a higher dropout rate during treatment $(5.0 \%$ vs. $2.7 \%)$ and more treatment failures (2.4\% vs. $1.7 \%$ ) but fewer recurrences (7.1\% vs. $14.6 \%)$. There was no evidence of increased risks of prolongation of the QT interval or dysglycemia with the 4-month regimen.

\section{CONCLUSIONS}

Noninferiority of the 4-month regimen to the standard regimen with respect to the primary efficacy end point was not shown. (Funded by the Special Program for Research and Training in Tropical Diseases and others; ClinicalTrials.gov number, NCT00216385.) 
HORTENED ANTITUBERCULOSIS TREATment regimens are expected to improve patient adherence to treatment, thus favoring better case management and disease control and minimizing the risk of drug resistance. ${ }^{1-3}$ The first indication that fluoroquinolones had the potential to shorten tuberculosis treatment was from an observational study in India ${ }^{4}$ in which ethambutol was replaced with ofloxacin. The fourth-generation fluoroquinolones gatifloxacin and moxifloxacin have shown mycobactericidal activity that is better than that of ofloxacin in vitro $^{5}$ and in vivo, ${ }^{6-9}$ and these agents have the potential to shorten treatment. Gatifloxacin was chosen for this study on the basis of its bactericidal-activity profile, cost, and generic status. We conducted a phase 3 trial that evaluated the efficacy and safety of a 4-month gatifloxacincontaining regimen, as compared with a standard 6-month regimen, for the treatment of rifampinsensitive pulmonary tuberculosis.

\section{METHODS}

\section{STUDY POPULATION}

Patients were recruited from cities in five subSaharan African countries: Cotonou in Benin, Conakry in Guinea, Nairobi in Kenya, Dakar in Senegal, and Durban in South Africa. Eligible patients were 18 to 65 years of age, with newly diagnosed, microbiologically confirmed pulmonary tuberculosis, as determined by the presence of acid-fast bacilli in two consecutive sputum smears; all patients provided written informed consent. The main exclusion criteria were a history of tuberculosis treatment during the previous 3 years, a history of insulin-dependent or noninsulin-dependent diabetes mellitus requiring treatment (because of potential glycemic imbalance in persons with tuberculosis), concomitant infection requiring additional anti-infective treatment, and human immunodeficiency virus (HIV) infection with World Health Organization (WHO) stage 3 disease (unless loss of $>10 \%$ of body weight was the only criterion met) or stage 4 disease. For a full list of exclusion criteria, see the study protocol, available with the full text of this article at NEJM.org.

\section{STUDY DESIGN}

This was a randomized, open-label, noninferiority, multicenter, controlled trial with a nested pharmacokinetic and pharmacokinetic-pharma- codynamic study. ${ }^{10}$ Patients were randomly assigned, in a 1:1 ratio with stratification according to country, to either a gatifloxacin-containing regimen (experimental group) or the 6-month standard treatment (control group). ${ }^{11}$

The control regimen consisted of isoniazid, rifampin, pyrazinamide, and ethambutol during the intensive phase of treatment ( 2 months), followed by isoniazid and rifampin during the continuation phase (4 months). The dosage of isoniazid, rifampin, pyrazinamide, and ethambutol followed WHO guidelines. ${ }^{11}$ In the experimental regimen, gatifloxacin (400 $\mathrm{mg}$ once daily irrespective of body weight [standard dose for treatment of infections]) was substituted for ethambutol during the intensive phase ( 2 months) and was maintained along with isoniazid and rifampin during the continuation phase (2 months). ${ }^{10}$ (For details on the treatment regimens, see Section S2 in the Supplementary Appendix, available at NEJM.org.) Fixed-dose combination tablets of isoniazid-rifampin, isoniazid-rifampin-pyrazinamide, or isoniazid-rifampin-pyrazinamide-ethambutol were manufactured and provided by Lupin Pharmaceuticals (Mumbai, India) in compliance with international manufacturing practice standards. Lupin Pharmaceuticals had no role in the conduct of the trial, the analysis of the data, or the preparation of the manuscript. All trial drugs were administered orally under supervision 6 days a week during the intensive phase and were provided every 2 weeks thereafter. Adherence was assessed by a count of the tablets that remained in weekly treatment boxes.

Clinical and laboratory evaluations were performed at baseline (before treatment), every month during the treatment phase, and every 2 or 3 months after the end of treatment for a total follow-up period of 24 months. Two sputum samples were obtained for direct smear examination, culture (solid medium), and drug-sensitivity tests at baseline and at all subsequent visits (Section S3 in the Supplementary Appendix). Initially, blood glucose was measured at 4 weeks and 8 weeks after the first drug intake and at the end of treatment; subsequently, on the advice of the data and safety monitoring board, blood glucose was measured more frequently (at 4 hours and 1,2 , and 12 weeks after the first drug intake). We obtained 12-lead electrocardiograms at baseline and between 1 and 5 hours after drug intake at 4 weeks, 8 weeks, and the end of treatment. (A timetable of all evaluations is provided 
in the protocol.) The trial was conducted in compliance with Good Clinical Practice and Good Clinical Laboratory Practice guidelines. Qualitycontrol and quality-assurance procedures were in place, including external quality control of microbiologic results by the Institute of Tropical Medicine, in Antwerp, Belgium; regular on-site monitoring visits with source-data verification for $100 \%$ of the data collected; and clinical audits of the trial sites (Section S4 in the Supplementary Appendix).

\section{STUDY OVERSIGHT}

The data and safety monitoring board reviewed trial data twice a year, with prespecified interim reviews of efficacy and safety. The protocol was approved by the national regulatory authorities in the participating countries and by the institutional review board or independent ethics committee at each participating site. The authors vouch for the completeness and accuracy of the data presented and for the fidelity of the study to the protocol.

\section{END POINTS}

The primary efficacy end point was the percentage of participants with an unfavorable outcome by 24 months after the end of treatment. This composite outcome included treatment failure (at either 4 months or 6 months after randomization, depending on the treatment group), recurrence (relapse or reinfection), and death or withdrawal from the study during the treatment phase. Treatment failure and recurrence were defined as positive results of two consecutive sputum cultures at least 1 day apart. When the outcome could not be determined, the case was submitted to an independent end-points committee, whose members were unaware of the study-group assignments. Participants with negative cultures at the end of follow-up were considered to have had a favorable outcome.

Secondary efficacy end points and safety end points included smear and culture conversion at 8 weeks, an unfavorable outcome at the end of treatment (treatment failure, death, or dropout), recurrence, an unfavorable outcome 18 months after randomization, the time to an unfavorable outcome by 24 months after the end of treatment, serious adverse events, hyperglycemia during the treatment phase, and prolongation of the QT interval (corrected QT [QTc] interval, calcu- lated with Fridericia's formula or Bazett's formula, of $>480 \mathrm{msec})^{12,13}$

\section{STATISTICAL ANALYSIS}

In the initial sample-size calculation, recurrence was used as the primary outcome (Section S1 in the Supplementary Appendix). The calculation was subsequently revised to allow for the abovementioned composite end point as the primary outcome..$^{10}$ The sample size was based on a onesided formula ${ }^{14}$ for equivalence, because the risk of an unfavorable outcome in the experimental group was not expected to be less than that in the control group. Assuming a $20 \%$ risk of an unfavorable outcome at 24 months after the end of treatment, we calculated that with 697 patients in each group, the study would have $80 \%$ power to show noninferiority of the experimental regimen versus the control regimen with a margin of 6 percentage points at a one-sided level of significance of 0.025 . To ensure that the study would be powered for both the intention-to-treat and perprotocol analyses and assuming that we would not be able to assess the primary outcome for $15 \%$ of the patients, we calculated that $820 \mathrm{pa}-$ tients had to be enrolled in each group (1640 in total). ${ }^{10}$

For all binary efficacy end points, we calculated the absolute difference in the proportion of patients with an unfavorable outcome, with adjustment for country, in the modified intentionto-treat and per-protocol populations. The modified intention-to-treat population consisted of all patients who underwent randomization and received the study medication at least once, excluding patients with suspected or confirmed rifampin-resistant tuberculosis. All patients included in the modified intention-to-treat analysis who missed no more than two consecutive doses during the intensive phase of treatment or no more than six consecutive doses during the continuation phase and who took at least $80 \%$ of all doses over a period of 4 months (experimental group) or 6 months (control group) were included in the per-protocol analysis. The time to an unfavorable outcome and the time to recurrence were analyzed with the use of Cox proportionalhazards regression (Section S5 in the Supplementary Appendix).

Prespecified subgroup analyses of the primary end point were performed according to country, HIV status, status with respect to iso- 
niazid resistance, and extent of pulmonary disease (on the basis of the zone score, which ranges from 0 to 6 , with 0 indicating that no lung regions are affected and 6 indicating that all lung regions are affected). Additional post hoc subgroup analyses were performed according to a body-mass index (BMI, the weight in kilograms divided by the square of the height in meters) of less than 16 (severe undernourishment) versus 16 or more ${ }^{15}$ and status with respect to cavitary disease. ${ }^{16}$

Safety analyses were conducted in the intention-to-treat population. Serious adverse events, prolongation of the QTc interval, and dysglycemia are reported in this article.

\section{RESULTS}

BASELINE CHARACTERISTICS OF THE PATIENTS

From June 2005 through October 2009, a total of 1836 patients were enrolled: 316 in Benin, 452 in Guinea, 200 in Kenya, 358 in Senegal, and 510 in South Africa (Fig. 1). The number of patients assigned to each group and the exclusions at each stage were well balanced, except in the per-protocol population, owing to a higher dropout rate during treatment in the control group than in the experimental group. All 138 patients enrolled in one center were excluded owing to evidence of fraudulent conduct.

The baseline characteristics of the patients were similar in the two treatment groups (Table 1, and Tables S1 and S2 in the Supplementary Appendix). Approximately $18 \%$ of the participants were HIV-positive. Half the participants presented with advanced pulmonary tuberculosis: $51 \%$ had a smear reading of $3+, 51 \%$ had cavitary pulmonary tuberculosis, and $44 \%$ had a zone score of 4 to 6 on chest radiography.

\section{EFFICACY}

\section{Primary Efficacy End Point}

A total of 1356 patients (694 in the experimental group and 662 in the control group) were included in the modified intention-to-treat analysis of the primary efficacy end point (Table 2). Overall, the country-adjusted difference (experimental group minus control group) in the rate of unfavorable outcome was 3.5 percentage points, with an upper boundary of the one-sided $97.5 \%$ confidence interval (CI) of 7.7. In the per-protocol analysis (Table 2), the difference between groups was slightly larger in favor of the control group (adjusted difference, 5.5 percentage points; upper boundary of the one-sided $97.5 \%$ CI, 9.4). Results were consistent for the time to an unfavorable outcome, with a country-adjusted hazard ratio (experimental group vs. control group) of 1.31 (95\% CI, 1.02 to 1.67 ) in the modified intentionto-treat population.

The reasons for unfavorable outcomes differed in the two treatment groups (Table 3, and Tables S3 and S4 in the Supplementary Appendix). More patients in the control group than in the experimental group dropped out during treatment $(5.0 \%$ vs. $2.7 \%)$ and had treatment failure ( $2.4 \%$ vs. $1.7 \%)$, whereas more patients in the experimental group than in the control group had a recurrence of tuberculosis $(14.6 \%$ vs. $7.1 \%)$.

The prespecified subgroup analysis according to country in the modified intention-to-treat population showed heterogeneity of treatment effect ( $\mathrm{P}=0.02$ for interaction between country and treatment group) (Fig. 2, and Table S5 in the Supplementary Appendix). Between-group differences in the rate of an unfavorable outcome ranged from -5.4 percentage points (Guinea) to 12.3 percentage points (Senegal).

Baseline HIV status, prevalence of cavitary pulmonary disease, and BMI varied among countries (Table S2 in the Supplementary Appendix). A prespecified subgroup analysis according to HIV status in the modified intentionto-treat population showed fewer unfavorable outcomes in the experimental group than in the control group among HIV-positive patients (difference in the rate of an unfavorable outcome, -4.0 percentage points; $95 \% \mathrm{CI},-15.7$ to 7.6 ; $\mathrm{P}=0.12$ for interaction) (Fig. 2, and Table $\mathrm{S} 5$ in the Supplementary Appendix). The variability of treatment effect according to country was further explored through post hoc subgroup analyses according to cavitary disease on chest radiography and BMI at baseline. The proportion of patients with an unfavorable outcome was similar in the two groups among those with noncavitary disease (difference, -1.3 percentage points; $95 \% \mathrm{CI},-7.3$ to 4.6 ; $\mathrm{P}=0.04$ for interaction) and among those with a BMI of less than 16 (difference, -0.4 percentage points; 95\% CI, -6.7 to $5.9 ; \mathrm{P}=0.10$ for interaction), but there were better outcomes in the control group than in the experimental group among patients with 


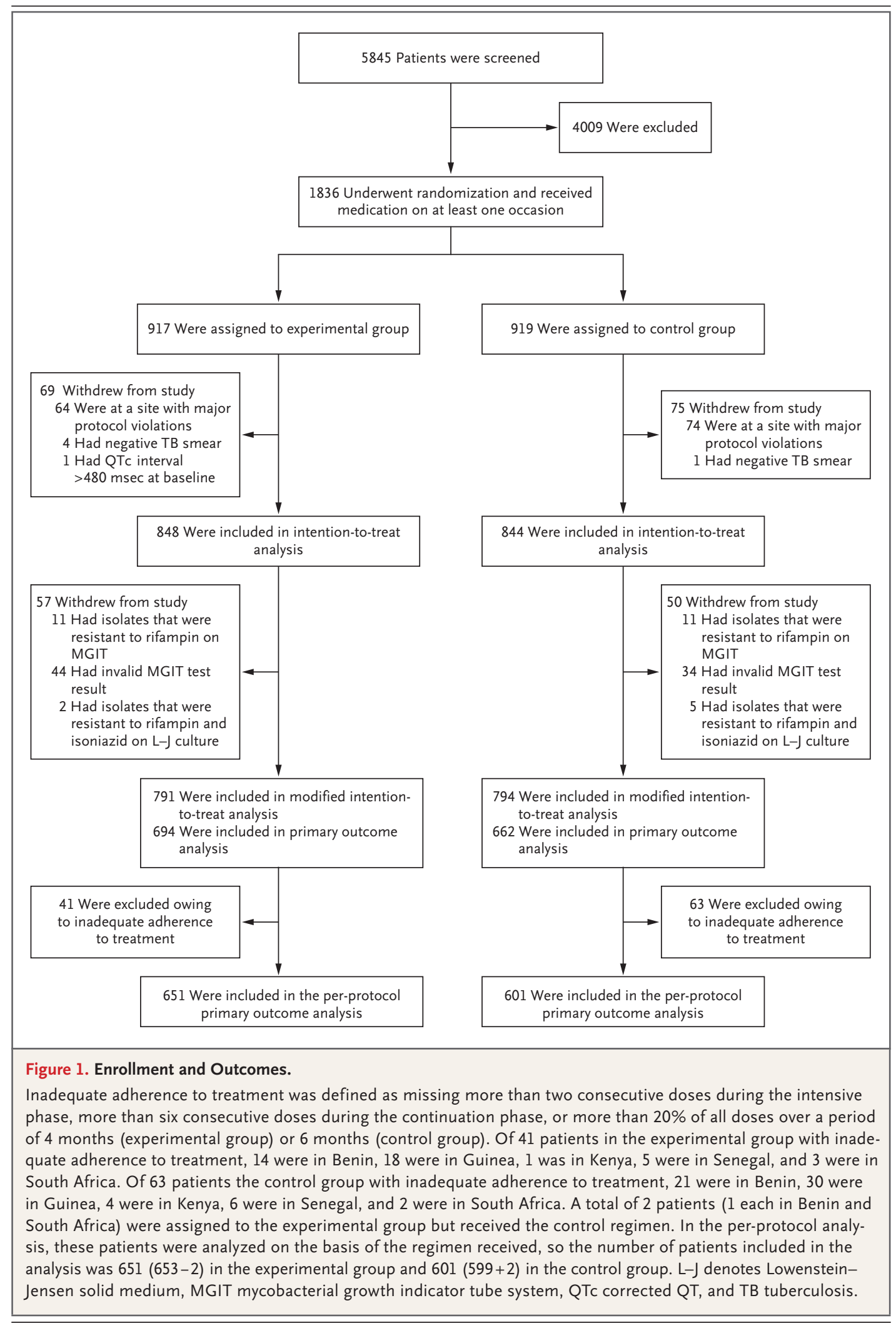


cavitary disease and among those with a BMI of 16 or more. There was no evidence of a significant interaction of treatment group with isoniazid resistance or with zone score on chest radiography at baseline ( $\mathrm{P}=0.47$ for both comparisons).

\section{Secondary Efficacy End Points}

Outcomes at 2 months after randomization and at the end of treatment were similar in the experimental and control groups. At 18 months after randomization, the experimental regimen was inferior to the control regimen in both the modified intention-to-treat and per-protocol populations (Table 2).

The country-adjusted hazard ratio (experimental group vs. control group) for the time to recurrence among those with a favorable outcome at the end of treatment was 2.25 (95\% CI, 1.59 to 3.18) (Table 2). There was strong evidence for nonproportional hazards with regard to treatment effect $(\mathrm{P}=0.004)$ : the country-adjusted hazard ratio up to 20 weeks after the end of treatment was 4.42 (95\% CI, 2.53 to 7.76 ), as compared with a hazard ratio of 1.22 (95\% CI, 0.74 to 2.01) at more than 20 weeks after the end of treatment (Fig. S1 in the Supplementary Appendix). Of 140 patients with culture-positive recurrence, $77(55 \%)$ had strains genotyped by means of a 15-locus mycobacterial interspersed repetitive unit-variable-number tandem-repeat analysis. Of these 77 patients, 15 of 20 patients in the experimental group (75\%) and 46 of 57 patients in the control group (81\%) had a relapse $(\mathrm{P}=0.75)$.

\section{SAFETY}

\section{Serious Adverse Events}

Overall, 43 serious adverse events were recorded in 43 patients; 5 of these events were judged to be related to the study medication. There were no significant differences between the groups with regard to the percentage of patients with at least one serious adverse event and the percentage of serious adverse events that were judged to be related to the study medication $(\mathrm{P}=0.65$ for both comparisons) (Tables S7, S8, and S9 in the Supplementary Appendix).

\section{QTc Interval}

There was no increased risk of an abnormal peak value of the QTc interval in the experimental
Table 1. Baseline Characteristics of Patients in the Intention-to-Treat Population.*

\begin{tabular}{|c|c|c|}
\hline Characteristic & $\begin{array}{l}\text { Experimental Group } \\
\qquad(\mathrm{N}=848)\end{array}$ & $\begin{array}{l}\text { Control Group } \\
(\mathrm{N}=844)\end{array}$ \\
\hline \multicolumn{3}{|l|}{ Country - no. (\%) } \\
\hline Benin & $158(18.6)$ & $158(18.7)$ \\
\hline Guinea & $221(26.1)$ & $225(26.7)$ \\
\hline Kenya & $100(11.8)$ & $100(11.8)$ \\
\hline Senegal & $178(21.0)$ & $180(21.3)$ \\
\hline South Africa & $191(22.5)$ & $181(21.4)$ \\
\hline Age $-y r$ & $30.9 \pm 9.1$ & $30.6 \pm 9.0$ \\
\hline Female sex — no. (\%) & $229(27.0)$ & $233(27.6)$ \\
\hline Body-mass index广 & $17.4 \pm 4.9$ & $17.5 \pm 5.0$ \\
\hline Weight — kg & $53.8 \pm 7.7$ & $54.2 \pm 8.0$ \\
\hline HIV positivity — no./total no. (\%) & $147 / 843(17.4)$ & $157 / 838(18.7)$ \\
\hline \multicolumn{3}{|l|}{ Smear findings - no./total no. (\%) } \\
\hline $1+$ & $129 / 847(15.2)$ & $141 / 842(16.7)$ \\
\hline $2+$ & $273 / 847(32.2)$ & $287 / 842(34.1)$ \\
\hline $3+$ & $445 / 847(52.5)$ & $414 / 842(49.2)$ \\
\hline Positive culture - no./total no. (\%) & $824 / 838(98.3)$ & $821 / 833(98.6)$ \\
\hline Isoniazid resistance — no./total no. (\%) & $41 / 617(6.6)$ & $53 / 622(8.5)$ \\
\hline Cavitation — no./total no. (\%) & $440 / 845(52.1)$ & $417 / 837(49.8)$ \\
\hline Bilateral lung disease — no./total no. (\%) & $508 / 838(60.6)$ & $523 / 832(62.9)$ \\
\hline Zone score of 4 to 6 - no./total no. (\%) $\mathbb{S}$ & $366 / 841(43.5)$ & $363 / 833(43.6)$ \\
\hline Corrected QT interval — msec $\mathbb{q}$ & $415.1 \pm 22.7$ & $414.7 \pm 25.8$ \\
\hline
\end{tabular}

* Plus-minus values are means \pm SD. Patients who could not be assessed for the efficacy outcomes were excluded from the analysis. HIV denotes human immunodeficiency virus.

$\uparrow$ The body-mass index is the weight in kilograms divided by the square of the height in meters.

$\ddagger$ Smear findings were scored $1+$ to $3+$, with higher numbers indicating more severe disease.

$\int$ The zone score ranges from 0 to 6 , with 0 indicating that no lung regions are affected and 6 indicating that all lung regions are affected.

I The QT interval was corrected for heart rate according to Bazett's formula.

group as compared with the control group. The risk difference (experimental group minus control group) was -0.4 percentage points with the QTc interval calculated with Bazett's formula ${ }^{12}$ and -0.9 percentage points with the interval calculated with Fridericia's formula ${ }^{13}$ (Table S10 in the Supplementary Appendix).

\section{Blood Glucose}

The vast majority of the patients had normal blood glucose levels throughout the course of treatment $(94.6 \%$ in the experimental group and 
Table 2. Primary and Secondary Efficacy Analyses in the Modified Intention-to-Treat Population and Per-Protocol Population.*

Variable

Experimental Group Control Group

Modified intention-to-treat analysis

Primary outcome: unfavorable outcome 24 mo after the end of treatment - no./total no. (\%)

Secondary outcomes

Smear-positive status at 2 mo - no./total no. (\%)

No culture conversion at 2 mo - no./total no. (\%)

Unfavorable outcome at end of treatment - no./ total no. (\%)

Unfavorable outcome 18 mo after randomization - no./total no. (\%)

No unfavorable outcome by 24 mo after the end of treatment - cumulative $\%$

No recurrence by 24 mo after the end of treatment - cumulative \%

\section{Per-protocol analysis}

Primary outcome: unfavorable outcome 24 mo after the end of treatment - no./total no. (\%)

Secondary outcomes

Smear-positive status at 2 mo - no./total no. (\%)

No culture conversion at 2 mo - no./total no. (\%)

Unfavorable outcome at end of treatment - no./ total no. (\%)

Unfavorable outcome 18 mo after randomization - no./total no. (\%)

No unfavorable outcome by 24 mo after the end of treatment - cumulative $\%$

No recurrence by 24 mo after the end of treatment - cumulative \%

$108 / 687(15.7)$
Adjusted Difference, Experimental GroupControl Group'广

percentage points (95\% Cl)

\section{$146 / 694(21.0)$}

$114 / 662(17.2)$

$3.5(-0.7$ to 7.7$)$

$118 / 762(15.5)$

$112 / 759$ (14.8)

0.0 (-3.0 to 2.9$)$

104/741 (14.0)

$121 / 735(16.5)$

$-2.0(-4.9$ to 1.0$)$

45/781 (5.8)

$67 / 785$ (8.5)

$-2.4(-4.9$ to 0.0$)$

139/731 (19.0)

98/744 (13.2)

5.8 (2.0 to 9.6$)$

80.7

85.5

1.31 (1.02 to 1.67$) \rrbracket$

85.6

93.5

2.25 (1.59 to 3.18$) \mathbb{\int}$

$115 / 651$ (17.7)

68/601 (11.3)

5.5 (1.6 to 9.4$)$

$118 / 752(15.7)$

$111 / 750$ (14.8)

$0.0(-2.9$ to 3.0$)$

103/732 (14.1)

$121 / 726$ (16.7)

$-1.9(-4.9$ to 1.1$)$

$17 / 736$ (2.3)

24/716 (3.4)

$-1.0(-2.7$ to 0.7$) \mid$

$53 / 676(7.8)$

7.3 (3.9 to 10.7)

83.7

90.6

1.73 (1.28 to 2.33$)$ d

85.7

93.7

2.32 (1.63 to 3.32 ) $\mathbb{8}$

* P values for interaction of treatment with country in the modified intention-to-treat population were as follows: end of treatment, $0.10 ; 18$ months after randomization, 0.04; 24 months after the end of treatment, 0.02 ; time to an unfavorable outcome, 0.04 ; and time to recurrence, 0.34 . P values for country interaction in the per-protocol population were as follows: end of treatment, not assessed; 18 months after randomization, $0.05 ; 24$ months after the end of treatment, 0.04; time to an unfavorable outcome, 0.12 ; and time to recurrence, 0.65 . Cl denotes confidence interval.

$\uparrow$ Differences were adjusted according to country unless otherwise indicated.

$\$$ The number of patients included in the analysis was 782 in the experimental group and 785 in the control group in the modified intention-to-treat population and 736 in the experimental group and 716 in the control group in the per-protocol population. $\int$ Shown is the hazard ratio, with $95 \% \mathrm{Cl}$, for the experimental regimen versus the standard regimen.

I The number of patients included in the analysis was 727 in the experimental group and 710 in the control group in the modified intention-to-treat population and 709 in the experimental group and 684 in the control group in the per-protocol population. || The difference was not adjusted according to country (model failed to converge).

$94.2 \%$ in the control group). Four patients, two in each study group, had grade 4 hyperglycemia (blood glucose $>27.5 \mathrm{mmol}$ per liter). No signifi- cant between-group difference was detected in the risk of hyperglycemia (Table S11 in the Supplementary Appendix). 
Table 3. Percentages of Favorable and Unfavorable Outcomes in the Primary Efficacy Analysis and of Outcomes That Could Not Be Assessed in the Modified Intention-to-Treat Population.

\begin{tabular}{lcc} 
Variable & Experimental Group & Control Group \\
Favorable outcome* & $548(79.0)$ & $548(82.8)$ \\
Unfavorable outcome* & $146(21.0)$ & $114(17.2)$ \\
By end of treatment & $45(6.5)$ & $67(10.1)$ \\
$\quad$ Study dropout & $19(2.7)$ & $33(5.0) \dagger$ \\
$\quad$ Withdrawal of consent & $8(1.2)$ & $8(1.2)$ \\
$\quad$ Adverse event other than death & $1(0.1)$ & $1(0.2)$ \\
$\quad$ Death & $5(0.7)$ & $9(1.4) \div$ \\
$\quad$ Treatment failure & $12(1.7)$ & $16(2.4)$ \\
After end of treatment: recurrence of tuberculosis & $101(14.6)$ & $47(7.1)$ \\
$\quad$ Two positive cultures & $86(12.4)$ & $33(5.0)$ \\
$\quad$ One positive culture & $12(1.7)$ & $9(1.4)$ \\
$\quad$ Culture-negative or unknown status $\mathbb{1}$ & $3(0.4)$ & $5(0.8)$ \\
Outcome could not be assessed & $97(12.3)$ & $132(16.6)$ \\
Protocol-defined withdrawal $\|$ & $8(1.0)$ & $8(1.0)$ \\
Loss to follow-up after end of treatment*** & $68(8.6)$ & $104(13.1)$ \\
Death after end of treatment & $19(2.4)$ & $18(2.3)$ \\
Withdrawal of consent after end of treatment & $1(0.1)$ & $1(0.1)$ \\
Other reason & $1(0.1)$ & $1(0.1)$ \\
\hline
\end{tabular}

* The denominator used to calculate the percentages for favorable outcome and unfavorable outcome was the total number of patients for whom the outcome could be assessed: 694 patients in the experimental group and 662 patients in the control group.

$\dagger \quad$ Of the 33 patients in the control group who dropped out during treatment, 26 (79\%) dropped out during the first 4 months of treatment and $7(21 \%)$ dropped out during months 5 and 6.

$¥$ Of the 9 patients in the control group who died during treatment, 7 died during the first 4 months of treatment and 2 died during months 5 and 6 .

$\int$ In these patients, recurrence was diagnosed on the basis of positive smears or symptoms of tuberculosis.

I The denominator used to calculate the percentages for Outcome could not be assessed was the number of patients eligible for the modified intention-to-treat population: 791 patients in the experimental group and 794 patients in the control group.

|| A total of 16 patients were withdrawn from the study during treatment because they started antiretroviral therapy ( 6 patients in the experimental group and 3 patients in the control group) or became pregnant ( 2 patients in the experimental group and 5 patients in the control group).

$* *$ The cumulative percentage of patients retained in the experimental and control groups, respectively, was $93.5 \%$ and $93.4 \%$ by 52 weeks, $91.0 \%$ and $89.6 \%$ by 78 weeks, and $87.5 \%$ and $82.7 \%$ by 94 weeks.

\section{DISCUSSION}

This trial assessed the effect of shortening the treatment duration for rifampin-sensitive tuberculosis by using a fluoroquinolone-based regimen. Despite a small absolute difference between regimens (3.5 percentage points) in the proportion of patients with an unfavorable outcome, the study failed to show that 4-month treatment with gatifloxacin substituted for ethambutol was noninferior to the 6-month standard regimen when a noninferiority margin of 6 percentage points was used. The results were consistent in the modified intention-to-treat and per-protocol analyses. The main reason for a failure to show noninferiority was the higher recurrence rate observed with the 4-month regimen, despite rapid culture conversion during treatment.

Treatment effects differed widely across countries and subgroups of patients. The risk of an unfavorable outcome with the 4-month regimen was almost twice as high in South Africa as in 


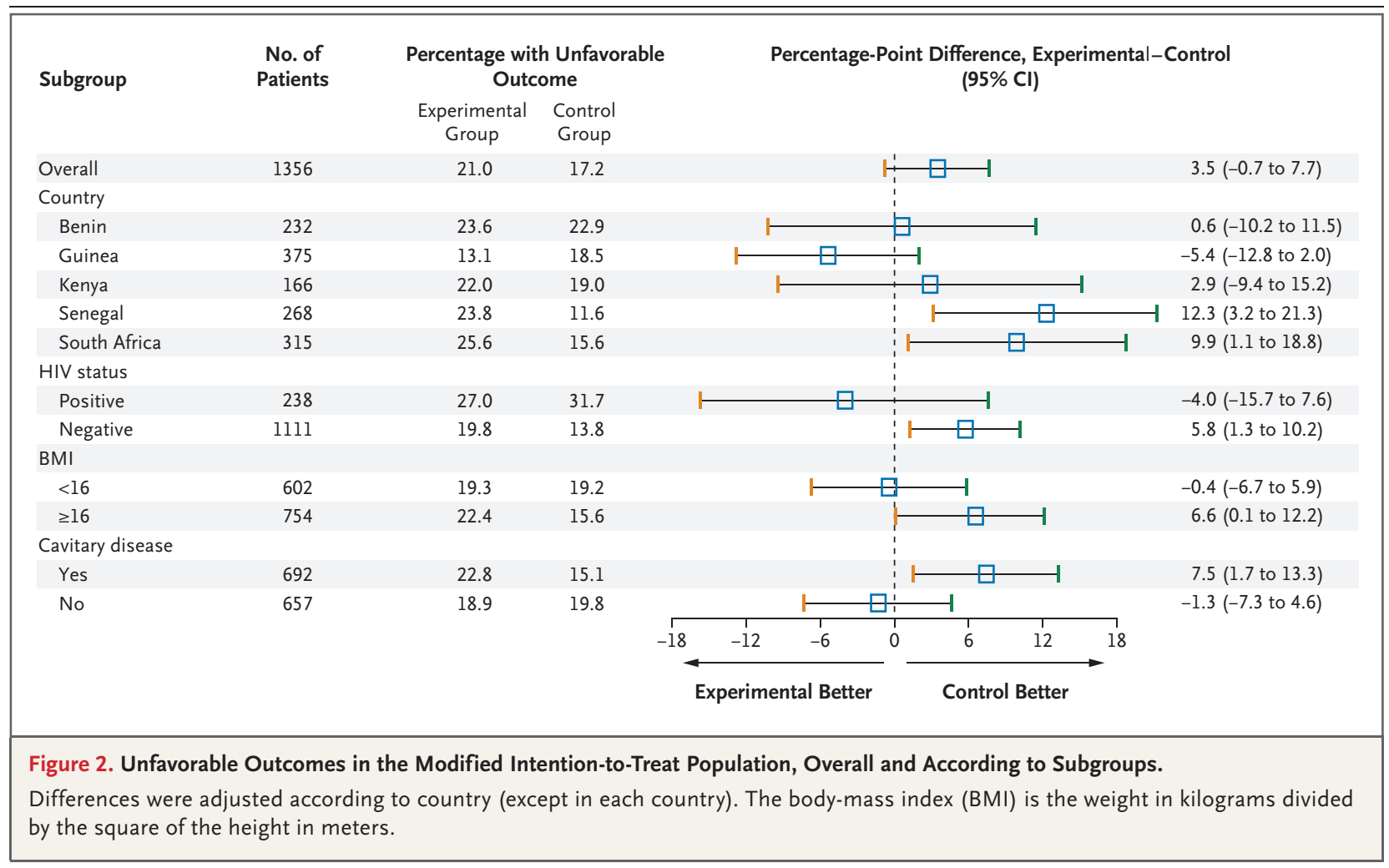

Guinea ( $25.6 \%$ vs. $13.1 \%$ ), with treatment effects (experimental group minus control group) of 9.9 percentage points and -5.4 percentage points, respectively. The use of a composite outcome and differences in patient characteristics at baseline might have contributed to the heterogeneity. The medications (which were produced according to Good Manufacturing Practice standards by the same manufacturer and adequately stored on site), the mycobacteriology laboratories (with procedures standardized across sites and quality control in place), and the open-label design of the study (with blinded outcome assessment by laboratory technicians and members of the endpoint committee $)^{10}$ are unlikely contributors.

This study used a composite end point that included events occurring during treatment (treatment failure, death, or dropout) and follow-up (recurrence). The contribution of these individual components to the overall end point varied among countries. Historically, a single outcome (cure without recurrence) was the primary efficacy end point ${ }^{17}$; now, however, a composite outcome is favored by regulators ${ }^{16}$ and is programmatically more relevant as a measure of true treatment effectiveness - although it is inherently more complex to interpret. Although more patients dropped out or died during treatment in the control group than in the experimental group (42 vs. 24), nearly $80 \%$ of the events in the control group occurred during the first 4 months of treatment, suggesting that the difference did not result from a longer risk period of treatment.

The baseline characteristics of the patients were similar in the two treatment groups but differed across countries: the proportion of severely undernourished patients was $50 \%$ in Guinea and Benin and $40 \%$ in the other countries, the prevalence of HIV positivity ranged from $1 \%$ in Senegal to $49 \%$ in South Africa, and the rate of cavitary disease was 20\% in Benin, Guinea, and Kenya and $90 \%$ in Senegal and South Africa (Table S2 in the Supplementary Appendix). Subgroup analyses therefore can help identify sources of heterogeneity; in patients who had noncavitary disease or were HIV-positive or severely undernourished, treatment effects were similar in the two groups, whereas in patients with cavitation or who were HIV-negative or had a BMI of more than 16, the 4-month regimen was inferior to standard treatment.

An inability to show noninferiority may be 
related to insufficient drug exposure (daily and total dose), because the dosage of gatifloxacin was not adjusted on the basis of body weight, and treatment was administered 6 days a week. Hence, total exposure to a 4-month gatifloxacin regimen might have been insufficient for sustained sterilization of the respiratory tract, especially in patients with cavitation or a higher (vs. lower) BMI, as suggested by some evidence of interaction between treatment and these factors with respect to the primary outcome. Furthermore, a population pharmacokinetic model based on the blood levels of a subgroup of these patients predicted that only $62 \%$ of the study population would have a target ratio of area under the curve to minimal inhibitory concentration of more than 125 at steady state. ${ }^{18}$ The results of the nested pharmacokinetic-pharmacodynamic analyses may provide additional insights.

In this study, a 4-month gatifloxacin-containing regimen did not carry an increased risk of dysglycemia, an adverse event that has been reported in older adults ${ }^{19}$ but has not been confirmed in other studies. ${ }^{20,21}$ In addition, there was no evidence of prolongation of the QT interval.

The expectations raised by the phase 2 study were not borne out in this phase 3 trial. The direction of the difference between fluoroquinolone-containing treatment and standard treatment in achieving culture conversion at 8 weeks consistently favored fluoroquinolone regimens in all the phase 2 studies published so far, ${ }^{6,7,9}$ although the difference reached statistical significance in only one study. ${ }^{8}$ The 8 -week outcome results of this phase 3 study are consistent with the phase 2 data but were not maintained during follow-up and could not reliably predict sterilizing activity and recurrence. Similar disappointing results were found by Jindani et al. ${ }^{22}$ in a phase 3 trial of a 4-month moxifloxacin-based regimen. More robust phase 2 surrogate markers of treatment efficacy are needed to select suitable regimens for shortening tuberculosis treatment that can be further assessed in phase 3 studies. It is possible that a 4-month treatment regimen may benefit a subgroup of patients with tuberculosis, such as those with noncavitary disease, as proposed by others ${ }^{23}$ and suggested by our results.

Drs. Sismanidis, Olliaro, and Lienhardt and Mr. Lapujade are currently staff members of the World Health Organization (WHO); the authors alone are responsible for the views expressed in this publication, and they do not necessarily represent the decisions, policy, or views of the WHO.

Supported by the Special Program for Research and Training in Tropical Diseases (TDR), a cosponsored program of the United Nations Children's Fund, the United Nations Development Program, the World Bank, and the WHO, and in part by the European Commission (International Cooperation with Developing Countries program). TDR funding was provided by governmental and cosponsor core contributions to TDR. Specific support was also provided by the U.S. Agency for International Development, the government of Japan, the Global Alliance for TB Drug Development, and the Li Ka Shing Foundation Global Health Programme at the University of Oxford. Lupin Pharmaceuticals, India, provided study drugs.

Disclosure forms provided by the authors are available with the full text of this article at NEJM.org.

We thank the study participants; the staff at clinical sites; the members of the data and safety monitoring committee (A. Nunn [chair], A. Mwinga, and P. Godfrey-Fausset); the members of the end-point committee (F.X. Blanc, P. Godfrey-Fausset, and C. Perronne); and A.C. Stona, D. Ciocan, and N. Oubaya for their contribution to the statistical analysis.

\section{REFERENCES}

1. Ma Z, Lienhardt C, McIlleron H, Nunn AJ, Wang X. Global tuberculosis drug development pipeline: the need and the reality. Lancet 2010;375:2100-9.

2. Global tuberculosis report 2013. Geneva: World Health Organization (http:/l www.who.int/tb/publications/global_ report/en).

3. Gospodarevskaya E, Tulloch $\mathrm{O}$, Bunga $\mathrm{C}$, et al. Patient costs during tuberculosis treatment in Bangladesh and Tanzania: the potential of shorter regimens. Int J Tuberc Lung Dis 2014;18:810-7.

4. A randomised clinical trial to trial the efficacy of intermittent regimens containing ofloxacin in the treatment of smear positive pulmonary tuberculosis: annual report 2001-2002. Tamil Nadu, India: Tuberculosis Research Center.

5. Sulochana S, Rahman F, Paramasivan $\mathrm{CN}$. In vitro activity of fluoroquinolones against Mycobacterium tuberculosis. J Chemother 2005;17:169-73.

6. Rustomjee R, Lienhardt C, Kanyok T, et al. A phase II study of the sterilising activities of ofloxacin, gatifloxacin and moxifloxacin in pulmonary tuberculosis. Int J Tuberc Lung Dis 2008;12:128-38.

7. Burman WJ, Goldberg S, Johnson JL, et al. Moxifloxacin versus ethambutol in the first 2 months of treatment for pulmonary tuberculosis. Am J Respir Crit Care Med 2006;174:331-8.

8. Conde $\mathrm{MB}$, Efron $\mathrm{A}$, Loredo $\mathrm{C}$, et al. Moxifloxacin versus ethambutol in the initial treatment of tuberculosis: a doubleblind, randomised, controlled phase II trial. Lancet 2009;373:1183-9.

9. Dorman SE, Johnson JL, Goldberg S, et al. Substitution of moxifloxacin for isoniazid during intensive phase treatment of pulmonary tuberculosis. Am J
Respir Crit Care Med 2009;180:27380.

10. Merle CS, Sismanidis C, Sow OB, et al. A pivotal registration phase III, multicenter, randomized tuberculosis controlled trial: design issues and lessons learnt from the Gatifloxacin for TB (OFLOTUB) project. Trials 2012;13:61.

11. Treatment of tuberculosis: guidelines for national programmes. 3rd ed., rev. Geneva: World Health Organization, June 2004.

12. Bazett HC. An analysis of the timerelations of electrocardiograms. Heart 1920;7:353-70.

13. Fridericia LS. The duration of systole in the electrocardiogram of normal subjects and of patients with heart disease. Acta Med Scand 1920;53:469-86.

14. Jones B, Jarvis P, Lewis JA, Ebbutt AF. Trials to assess equivalence: the impor- 
tance of rigorous methods. BMJ 1996;313: 36-9. [Erratum, BMJ 1996;313:550.]

15. BMI classification. Geneva: World Health Organization (http://apps.who.int/ bmi/index.jsp?introPage=intro_3.html). 16. Guidance for industry - pulmonary tuberculosis: developing drugs for treatment. Rockville, MD: Food and Drug Administration, 2013 (http://www.fda.gov/ downloads/Drugs/GuidanceCompliance RegulatoryInformation/Guidances/ UCM373580.pdf).

17. Fox W, Ellard GA, Mitchison DA. Studies on the treatment of tuberculosis undertaken by the British Medical Research Council tuberculosis units, 1946-1986, with relevant subsequent publications. Int J Tuberc Lung Dis 1999;3:Suppl 2:S231-S279.
18. Smythe W, Merle CS, Rustomjee R, et al. Evaluation of initial and steadystate gatifloxacin pharmacokinetics and dose in pulmonary tuberculosis patients by using Monte Carlo simulations. Antimicrob Agents Chemother 2013;57:416471.

19. Park-Wyllie LY, Juurlink DN, Kopp A, et al. Outpatient gatifloxacin therapy and dysglycemia in older adults. N Engl J Med 2006;354:1352-61.

20. Mohr JF, McKinnon PS, Peymann PJ, Kenton I, Septimus E, Okhuysen PC. A retrospective, comparative evaluation of dysglycemias in hospitalized patients receiving gatifloxacin, levofloxacin, ciprofloxacin, or ceftriaxone. Pharmacotherapy 2005;25:1303-9.
21. Onyenwenyi AJ, Winterstein AG, Hatton RC. An evaluation of the effects of gatifloxacin on glucose homeostasis. Pharm World Sci 2008;30:544-9.

22. Jindani A, Hatherill $M$, Charalambous S, et al. A multicentre randomized clinical trial to evaluate high-dose rifapentine with a quinolone for treatment of pulmonary TB: the RIFAQUIN Trial. Presented at the 20th Conference on Retroviruses and Opportunistic Infections, Atlanta, March 3-6, 2013. abstract.

23. Phillips PP, Nunn AJ, Paton NI. Is a 4-month regimen adequate to cure patients with non-cavitary tuberculosis and negative cultures at 2 months? Int J Tuberc Lung Dis 2013;17:807-9.

Copyright $\odot 2014$ Massachusetts Medical Society. 